\title{
Reproductive outcomes following robotic myomectomy
}

\author{
Kathryn L. Shaia*, Lucky H. Sekhon, Leigh Rosen, Alicia Robbins, \\ Nicole Astill, Charles Ascher-Walsh
}

Department of Obstetrics, Gynecology and Reproductive Science, Icahn School of Medicine at Mount Sinai, New York, USA

Received: 17 January 2017

Accepted: 09 March 2017

*Correspondence:

Dr. Kathryn L. Shaia,

E-mail: kathryn.shaia@Mountsinai.org

Copyright: (c) the author(s), publisher and licensee Medip Academy. This is an open-access article distributed under the terms of the Creative Commons Attribution Non-Commercial License, which permits unrestricted non-commercial use, distribution, and reproduction in any medium, provided the original work is properly cited.

\section{ABSTRACT}

Background: To assess pregnancy outcomes of patients following robotic myomectomy.

Methods: Retrospective chart review was performed of 336 patients following robotic myomectomy at a university hospital from June 2006 to May 2013. Patients were called to obtain delivery outcomes.

Results: Three hundred and thirty-six women had the following: mean age of $41.97+/-12.3$ years (range 24-55), mean BMI of 24.6 +/- 4.96, mean of 3.37 +/- 3.1 fibroids removed (range 1-21), and mean weight of $352.28+/$ 339.56 grams of fibroids removed. Approximately $66.9 \%(\mathrm{~N}=250)$ provided pregnancy outcome data with 119 (47.6\%) attempting pregnancy after surgery. Eighty-three (69.7\%) achieved a total of 91 pregnancies. Less than half of these patients underwent cesarean section, and no cases of uterine rupture. Eighty-seven patients had known infertility pre-surgery; 22 underwent intrauterine insemination resulting in 12 pregnancies, and 34 underwent in vitro fertilization resulting in 25 pregnancies. A total of $53(60.9 \%)$ of patients with infertility achieved 60 total pregnancies.

Conclusions: The fertility rate after robotic myomectomy for patients attempting to conceive (69.7\%) and for those with known infertility who continued to attempt pregnancy postoperatively (60.9\%) were similar. There was a low incidence of complications associated with pregnancies conceived after robotic myomectomy.

Keywords: Infertility, Leiomyoma, Robotics, Robotic surgical procedures, Uterine myomectomy

\section{INTRODUCTION}

Leiomyomas are the most common benign tumors among women and are found clinically in $25 \%$ of women, with some studies estimating a true prevalence of greater than $70 \% .^{1-4}$ Symptomatic fibroids serve as the most common indication of the approximately 540,000 hysterectomies performed annually in the United States. ${ }^{5}$ However, in reproductive-aged women who desire uterine conservation and fertility, myomectomy is the treatment of choice. ${ }^{5}$ Fibroids are estimated to be present in 5-10\% of women with subfertility and may act as the primary cause of infertility in $2-3 \%$ of cases. ${ }^{3,6,7}$
Fibroids are hypothesized to interfere with normal reproductive tract function and fertility via various mechanisms. Submucous fibroids that distort the contour of the uterine cavity may contribute to infertility and miscarriage. ${ }^{8-10}$ In cases where fibroids are intramural and do not distort the uterine cavity, published studies report inconsistent findings. ${ }^{5,8,11-15}$ A systematic review by Falcone et al. demonstrated that the presence of intramural fibroids reduced implantation, clinical pregnancy and live birth rates in a cohort of women with normal uterine cavities on hysterosalpingogram or saline sonogram. ${ }^{8}$

In carefully selected candidates, who have had a complete fertility evaluation to rule out other potential combined or 
isolated causal factors, surgical treatment of uterine fibroids may be indicated. ${ }^{16}$ While an open myomectomy may be the most effective treatment for patients with a large number of fibroids throughout the uterus, a minimally invasive approach is advantageous in patients with fewer fibroids or a large, solitary fibroid that requires abdominal access for removal. Laparoscopic myomectomy (LM) and robotic-assisted laparoscopic myomectomy (RALM) are minimally invasive procedures associated with less postoperative pain, lower postoperative fever, shorter hospital stay and quicker return to normal activity when compared to abdominal myomectomy. ${ }^{16-17}$ There has been no evidence suggesting a difference in recurrence risk between laparoscopic and open myomectomy. ${ }^{1}$ However, when pregnancy is desired, the technique appears particularly advantageous in that it could reduce the risk of postoperative adhesions compared with laparotomy. ${ }^{18-20}$

Uterine rupture and abnormal placentation have been cited as possible complications following myomectomy due to weakening of myometrial integrity. The rate of uterine rupture after abdominal myomectomy has been estimated as $<1 \%$ in most studies. ${ }^{21-22}$ The risk of uterine rupture has been reported to be slightly higher after a laparoscopic myomectomy with a meta-analysis of 56 articles reporting a slightly increased occurrence of uterine rupture after laparoscopic myomectomy (1.2\%) versus abdominal myomectomy $(0.4 \%){ }^{21}$ Other studies have demonstrated a 0 to $1 \%$ risk of uterine rupture following laparoscopic myomectomy. ${ }^{20,22-23}$ Similarly, the rate of uterine rupture following RALM has been reported anywhere between 0 to $1.1 \% .^{24,25}$

Since we first began to perform RALM in our institution, we have maintained a database concerning patient outcomes after undergoing this procedure. The objective of this study was to report post-operative fertility rates and pregnancy outcomes of a large series of patients who had a RALM with a single surgeon at Mount Sinai Hospital from June 2006 to May 2013.

\section{METHODS}

We conducted a retrospective chart review including 336 patients that underwent robotic myomectomy, with a single surgeon, at a large university hospital program. Standard office procedure included annual calls to all post-operative patients after myomectomy (see Appendix A for questionnaire) to determine whether they had become pregnant on one or more occasions since surgery, and the outcome of these pregnancies. Cases where patients were unable to be reached or they declined to participate were considered lost to follow up. All telephone interviews were recorded in the current electronic medical record system at Mount Sinai and became part of the patient's permanent chart. The Mount Sinai Hospital IRB waived consent requirements as the data was de-identified and retrospective.

\section{RESULTS}

Three hundred and thirty-six women had a mean age of $41.97+/-12.3$ years (range 24-55) and a mean BMI of $24.6+/-4.96$. Patients had a mean of $3.37+/-3.1$ fibroids removed (range 1-21), with the average weight of fibroids removed $352.28+/-339.56$ grams. Of the patients contacted via telephone call, $66.9 \% \quad(\mathrm{~N}=250)$ were successfully reached and agreed to provide fertility and pregnancy outcome data. Follow-up from time of surgery to most recent charted data ranged from 10 months to 7.9 years.

Indications for RALM included dysfunctional uterine bleeding $(29.2 \%, \mathrm{~N}=73)$, pelvic pain or pressure $(19.6 \%$, $\mathrm{N}=49)$, infertility or recurrent pregnancy loss $(12.8 \%$, $\mathrm{N}=32$ ) and a combination of these factors $(59.6 \%$, $\mathrm{N}=149)$. Eighty-three percent $(\mathrm{N}=175)$ of women had significant to complete resolution of symptoms after RALM with $58.9 \%$ reporting complete improvement. Eight patients $(3.2 \%)$ reported some recurrence of symptoms after the procedure, with time of recurrence ranging from 6 months to 2 years. However, no patients required an additional procedure for their fibroids over the follow up period.

Of the 250 women who were included in our study, 119 $(47.6 \%)$ actively tried for pregnancy after surgery. They began attempting to conceive on average 7.1 months post-operatively. Eighty-three $(69.7 \%)$ achieved a total of 91 pregnancies- 36 full term, 12 preterm, 23 spontaneous abortions, 19 ongoing pregnancies and 1 termination. Forty-six percent $(\mathrm{N}=43)$ of these patients underwent cesarean section, the majority of which were elective or recommended by their obstetrician due to their history of myomectomy. Of the women attempting to conceive after RALM, there was a subset of $87(73.1 \%)$ women with a known diagnosis of infertility prior to surgery. Twentytwo of these patients underwent intrauterine insemination (IUI) resulting in 12 pregnancies, while 34 underwent in vitro fertilization (IVF) culminating in 25 pregnancies. After surgery, a total of $52(60.0 \%)$ of patients with a diagnosis of infertility achieved pregnancy- 60 total pregnancies- 22 full term, 3 preterm, 15 spontaneous abortions, 11 ongoing pregnancies and 1 termination. Among the 250 cases, there was one pregnancy complicated by a cesarean-hysterectomy secondary to a placenta accreta. There were no reported cases of uterine rupture.

\section{DISCUSSION}

Fibroids are known to contribute to pregnancy complications and infertility in reproductive aged women. Myoma-related infertility is thought to be mediated by uterine contraction, which interferes with sperm migration and ovum transport, or enlarging the anatomical uterine cavity and secreting inflammatory and vasoactive substances, both of which may cause implantation failure. ${ }^{26} \mathrm{~A}$ myomectomy is indicated for 
patients with infertility or recurrent pregnancy loss with submucosal or cavity-distorting myomas. A prospective study of 46 women with myomas who underwent 172 IVF cycles showed no difference in implantation or pregnancy rates compared to 50 control patients undergoing 127 cycles unless the uterine cavity itself was distorted by the myomas. ${ }^{27}$ For fibroids that require an abdominal approach, RALM addresses some of the reproductive and obstetrical pitfalls of traditional laparotomy (minimally invasive) and laparoscopy (ease of suturing, facilitating multi-layer closure).

This is one of the largest series reporting on postoperative fertility and obstetric outcome after RALM to date. The overall pregnancy rate was reported as $69.7 \%$. This finding is in agreement with the postoperative pregnancy rates quoted in prior studies. Cela et al. reported that $77.8 \%$ of subjects desiring pregnancy were able to conceive and maintain a viable pregnancy after undergoing RALM. ${ }^{24}$ Lonnerfors and Persson reported that 15 out of $22(68.2 \%)$ women who actively attempted to achieve pregnancy were successful after removal of deep intramural myomas by RALM. ${ }^{28}$ Tusheva et al. reported a pregnancy rate of $75 \%$ in 16 RALM patients desiring pregnancy. ${ }^{29}$

Of the patients who conceived, the outcome of 72 pregnancies was known- $50.0 \% \quad(\mathrm{~N}=36)$ of which experienced a term delivery, $16.7 \%(\mathrm{~N}=12)$ delivered prior to 37 weeks gestation and $31.9 \% \quad(\mathrm{~N}=23)$ experienced early pregnancy loss. There is no evidence to support that gestational age at delivery is influenced by mode of myomectomy. A systematic review of two randomized control trials evaluating a total of 293 patients allocated to either open or laparoscopic myomectomy reported no significant effect on the live birth rate (OR $0.80,95 \%$ CI 0.42 to 1.50$)$, clinical pregnancy rate (OR $0.96,95 \%$ CI 0.52 to 1.78 ), ongoing pregnancy rate (OR $1.61,95 \%$ CI 0.26 to 10.04), miscarriage rate (OR $1.31,95 \%$ CI 0.40 to 4.27 ), or preterm labor rate (OR $0.68,95 \%$ CI 0.11 to 4.43$).{ }^{30}$ Recurrence of fibroids post-myomectomy has been associated with adverse obstetrical outcome. Sudik et al performed a comparative, retrospective non-randomized clinical study including 67 patients undergoing myomectomy for fertility and reported a higher rate of preterm delivery $(38.5 \%$ vs. $0 \%)$ and cesarean section $(84.6 \%$ vs. $38.8 \%)$ in patients with recurrent fibroids $(\mathrm{N}=31)$ compared with the recurrence-free control group $(\mathrm{N}=36) .{ }^{31}$ The rate of post-RALM early pregnancy loss reported in this study is not significantly increased from the incidence seen in reproductive aged women in the general population, reported anywhere between $10 \%$ to $30 \%$ or even higher in women with advanced maternal age. $^{32-33}$

Of the women attempting to conceive after RALM, there was a subset of 87 (out of 119) women with a known diagnosis of infertility prior to surgery. Fifty-six patients $(64.4 \%)$ relied on assisted reproduction in the form of either intrauterine insemination (IUI) or IVF in their attempt to conceive. The use of assisted reproduction to conceive post-myomectomy is widely reported in the literature. Pitter et al. conducted a multicenter retrospective analysis of the 872 women undergoing RALM over 5 years and reported that 107 subsequently conceived resulting in 127 pregnancies, 50 (39.3\%) of which were conceived with the aid of assisted reproduction, with IVF being the most common approach. ${ }^{34}$ In this large case series, $60.0 \%(\mathrm{~N}=52)$ of patients with infertility achieved pregnancy post-RALM. Twenty-two $(42.3 \%)$ of these patients underwent IUI resulting in 12 pregnancies, while $34(65.4 \%)$ underwent in IVF, leading to 25 pregnancies. Of all pregnancies achieved with a known outcome $(\mathrm{N}=49), 44.9 \%(\mathrm{~N}=22)$ were delivered at term gestation and $6.1 \%(\mathrm{~N}=3)$ were delivered prior to 37 weeks gestation. Thirty-one percent of pregnancies achieved $(\mathrm{N}=15)$ ended in early pregnancy loss. At the time of this study, $18.3 \%$ of pregnancies were ongoing. Thus, the postoperative obstetrical outcomes of infertility patients were similar to that seen in the general patient cohort who conceived after RALM.

In addition to fertility optimization, RALM also afforded significant to complete symptom relief in over $70 \%$ of all patients in our study, with the large majority remaining symptom-free years after surgery. ${ }^{34}$ No patients required a secondary procedure for their fibroids. Symptom relief has been shown to increase the chances of conception, with an $80 \%$ pregnancy rate for symptom-free patients after more than three-year follow-up. ${ }^{34}$

Among the 250 cases, there was one pregnancy complicated by a cesarean-hysterectomy secondary to a placenta accreta. There were no cases of uterine rupture. A retrospective cohort study analyzing 676 patients who underwent laparoscopic myomectomy reported an overall rate of placental abnormalities (including placenta previa, abruption, accreta and percreta) of $4.2 \%$ and an overall uterine rupture rate of $0.6 \% .^{35}$ There are at least two reports of uterine rupture during pregnancy following laparoscopic myomectomy, both at 34 weeks. ${ }^{18,36}$ Our data demonstrating that the risk of uterine rupture or major placental abnormalities after RALM is very low is in agreement with prior literature. Bannerman et al. analyzed registry data on patients who underwent RALM at various hospitals and reported that placenta accreta and uterine rupture occurred did not occur in any patients post-operatively. However, this finding is difficult to interpret as this may have been a mixed cohort of patients who underwent myomectomy via laparoscopy or laparotomy as the authors did not have access to any patients' operative reports and could not be sure of the exact procedure conducted $(\mathrm{N}=176) .{ }^{37}$ An inability to effectively close the myometrium laparoscopically could contribute to a higher incidence of these complications.

Patients attempted to conceive on average 7.1 months after the procedure, which is four months longer than the minimal interval of time often recommended to patients, 
based on the available literature. Findings from magnetic resonance imaging (MRI) studies after myomectomy suggests approximately 12 weeks preconception is required for uterine healing to occur and even longer if there is evidence of hematoma formation in the myometrium. ${ }^{38}$ Hence, a 12-week waiting period is suggested before attempting pregnancy. If there a significant myometrial defect is created during the procedure, cesarean delivery is often recommended with a view to reduce the risk of uterine rupture during labor and delivery. ${ }^{38}$

Of the patients who became pregnancy and delivered, $46.1 \%$ underwent cesarean section, the majority of which were elective or recommended by their obstetrician given their history of myomectomy. Prior studies have reported a post-RALM cesarean section rate of $95.7 \%$ and a wide range of cesarean section rates after laparoscopic myomectomy $(23.5 \%$ to $82.1 \%) .{ }^{25}$ A recent Cochrane Systematic Review reported no significant difference in the rate of cesarean section after open and laparoscopic myomectomy (OR 0.59 , 95\% CI 0.13 to 2.72 ). ${ }^{24}$ The considerably lower rate of cesarean section in our patients may have been influenced by the greater interval of time from the procedure and when they began actively attempting to conceive.

Studies indicate that RALM may obviate the need for laparotomy as it has shown to be a feasible approach for the removal of larger, more difficult myomas that are approached less often with traditional laparoscopic surgery. ${ }^{39}$ Compared to laparotomy, patients undergoing RALM have been shown to have a significantly less blood loss, a smaller change in postoperative hematocrit, and a shorter length of stay. ${ }^{17}$ Furthermore, RALM may avoid complications associated with myomectomy performed by laparotomy, including postoperative hemorrhage, ileus, bowel obstruction, cystotomy, and pelvic abscess formation. ${ }^{5}$ RALM has been shown to be associated with longer duration of surgery, ranging from 174.6 to 181 minutes in prior studies. ${ }^{31,39}$ Increased operative time is a major contributing factor to the increased cost of RALM. The average operating time for our patients undergoing RALM was 149 minutes. The cost of surgery can be minimized when performed by an experienced surgeon with decreased operative time and may be further offset by the decreased length of inpatient stay compared with laparotomy. ${ }^{39}$ Initial hospital charges have been found to be higher with lower physician reimbursement for robot-assisted surgery. ${ }^{17}$ However, as the costs secondary to depreciation of the robot decrease in combination with lower complication rates, length of stay, and nursing costs, robot-assisted technology may become the more optimal financial option. ${ }^{17}$

A strength of our study is that all cases were performed by a single surgeon using the same operative technique. In addition, our sample size was larger than most studies to date, reporting on the reproductive and pregnancy outcomes of 250 patients that underwent RALM. Our study was limited due to its retrospective design. Of the 336 patients we attempted to contact, $25.6 \%$ refused to participate or were lost to follow up. Of the patients interviewed, the data derived from the questionnaires may have been subject to recall bias. We were unable to account for each patient's fertility diagnosis, work up and treatment plan. Therefore, it is difficult to draw conclusions regarding which type of infertility patient and infertility treatment approach has the best outcome after RALM. The majority of patients in our study population had a normal BMI $(24.6+/-4.96)$ and were Caucasian $(54.3 \%)$ vs. $20 \%$ African-American, $24 \%$ Asian, and $12 \%$ Hispanic. This could potentially limit the generalizability of our findings to other patient populations. Differences in fibroid characteristics could have contributed to differences in fertility and pregnancy outcomes. We did not account for fibroid location or size. Myoma size has been shown to impact fertility and contribute to pregnancy loss. A retrospective study performed by Oliveira et al. studied 245 women with subserosal and/or intramural fibroids that did not compress the uterine cavity and demonstrated that intramural fibroids greater than $4.0 \mathrm{~cm}$ were associated with decreased pregnancy rates compared to patients with smaller fibroids $(29 \%$ vs. $53 \%, \mathrm{P}=0.025) .{ }^{15}$ Conversely, a retrospective cohort study by Zhang et al. analyzed the pregnancy live birth rate of 471 patients after either abdominal or laparoscopic myomectomy and reported that larger myomas (greater than $10 \mathrm{~cm}$ in size) may lead to earlier detection and intervention, allowing for better pregnancy outcomes. ${ }^{40}$ In addition to size, fibroid location is a major factor influencing clinical outcome after RALM. Elements such as precise dissection and suturing is challenging when the myomas have a deep intramural component or are located posteriorly or in the lower uterine segment. Unfortunately, these fibroid locations which may be difficult to access, especially laparoscopically, have a high propensity to affect fertility and obstetrical outcome. ${ }^{28}$

Major adverse obstetrical outcomes such as uterine rupture or placenta accrete are rare events and difficult to study retrospectively. Future studies in the form of randomized clinical trials are required to compare the efficacy, safety and cost-effectiveness of RALM, laparoscopic and open myomectomy for management of fibroids and the subsequent reproductive and obstetrical outcomes when pregnancy is achieved postoperatively. Future analyses should take into account these various potential confounding factors, accounting for fibroid size and location and patients' fertility status, diagnosis and post-RALM assisted reproduction treatment. Prospective studies should also focus on correlating the interval of time from RALM and conception with fertility and obstetrical outcome.

\section{CONCLUSION}

In conclusion, in reporting one of the largest series of patients who underwent RALM to date, we demonstrated 
that the pregnancy rate after robotic myomectomy for all women attempting to conceive $(69.7 \%)$ and for those with known infertility who continued to try for pregnancy postoperatively $(60.9 \%)$ were similar. This finding should reassure both patients and physicians regarding the efficacy of RALM for surgical management of fibroids in women who wish to preserve their fertility. The findings of this study demonstrated a very low incidence of major complications associated with the presence of uterine scarring in pregnancies conceived after RALM in both women with and without a diagnosis of fertility, over a 7year follow up period. With proper case selection, RALM may offer an appealing alternative to open or laparoscopic myomectomy to achieve optimal fertility and pregnancy outcomes for patients with fibroids.

\section{ACKNOWLEDGEMENTS}

This research was presented at the American Association of Gynecologic Laparoscopists $44^{\text {th }}$ Global Conference November 15-19, 2015 in Las Vegas, NV.

Funding: No funding sources

Conflict of interest: None declared

Ethical approval: Not required

\section{REFERENCES}

1. Chittawar P, Franik S, Pouwer AW. Minimally invasive surgical techniques versus open myomectomy for uterine fibroids. Cochrane Database of Systematic Reviews. 2014;10:CD004638.

2. Buttram VC, Reiter RC. Uterine leiomyomata. Fertil Steril. 1981;36:433-45.

3. Baird DD, Dunson DB, Hill MC. High cumulative incidence of uterine leiomyoma in black and white women: ultrasound evidence. Am J Obstet Gynecol. 2003;188:100-7.

4. Cramer SF, Patel A. The frequency of uterine leiomyomas. Am J Clin Pathol. 1990;94(4):435-8.

5. Falcone T, Parker WH. Surgical management of leiomyomas for fertility or uterine preservation. Obstet Gynecol. 2013;121:856-68.

6. Cardozo ER, Clark AD, Banks NK. The estimated annual cost of uterine leiomyomata in the United States. Am J Obstet Gynecol. 2012;206:211.

7. Practice Committee of the American Society for Reproductive Medicine. Myomas and reproductive function. Fertil Steril. 2006;86:194-99.

8. Pritts EA. Fibroids and infertility: a systematic review of the evidence. Obstet Gynecol Surv. 2001;58:483-91.

9. Heertum KV, Barmat L. Uterine fibroids associated with infertility. Women's Health 2014;10(6):645-53.

10. Brady PC, Stanic AK, Styer AK. Uterine fibroids and subfertility: an update on the role of myomectomy. Current Opinion in Obstetrics and Gynecology. 2013;25(3):255-9.
11. Casini ML, Rossi F, Agostini R. Effect of the position of fibroids on fertility. Gynecol Endocrinol. 2006;22:106-9.

12. Surrey ES, Lietz AK, Schoolcraft WB. Impact of intramural leiomyomata in patients with a normal endometrial cavity on in vitro fertilization-embryo transfer cycle outcome. Fertil Steril. 2001;75:405-10.

13. Rackow BW, Taylor HS. Submucosal uterine leiomyomas have a global effect on molecular determinants of endometrial receptivity. Fertil Steril. 2010;93:2027-34.

14. Sunkara SK, Khairy M, El-Toukhy T. The effect of intramural fibroids without uterine cavity involvement on the outcome of IVF treatment: a systematic review and meta-analysis. Hum Reproduction. 2010;25(2):418-29.

15. Oliveira, Flávio Garcia. Impact of subserosal and intramural uterine fibroids that do not distort the endometrial cavity on the outcome of in vitro fertilization-intracytoplasmic sperm injection. Fertil Steril. 2004;81(3):582-7.

16. Practice Committee of American Society for Reproductive Medicine in collaboration with Society of Reproductive Surgeons. Myomas and reproductive function. Fertil Steril. 2006;86:194-9.

17. Ascher-Walsh CJ, Capes TL. Robot-assisted laparoscopic myomectomy is an improvement over laparotomy in women with a limited number of myomas. J Minim Invasive Gynecol. 2010;17:30610.

18. Dubuisson JB, Chavet X, Chapron C. Uterine rupture during pregnancy after laparoscopic myomectomy. Hum Reprod. 1995;10:1475-7.

19. Bulletti C, Polli V, Negrini V. Adhesion formation after laparoscopic myomectomy. J Am Assoc Gynecol Laparosc. 1996;(3)533-6.

20. Dubuisson JB, Fauconnier A, Deffarges JV. Pregnancy outcome and deliveries following laparoscopic myomectomy. Hum Reprod. 2000;15(4)869-73.

21. Claeys J, Hellendoorn I, Hamerlynck T. The risk of uterine rupture after myomectomy: a systematic review of the literature and meta-analysis. Gynecol Surg. 2014;11:197-206.

22. Seracchioli R, Manuzzi L, Vianello F. Obstetric and delivery outcome of pregnancies achieved after laparoscopic myomectomy. Fertil Steril. 2006;86:159-65.

23. Sizzi O, Rossetti A, Malzoni M. Italian multicenter study on complications of laparoscopic myomectomy. J Minim Invasive Gynecol. 2007; 14:453-62.

24. Cela V, Freschi L, Simi G. Fertility and endocrine outcome after robot-assisted laparoscopic myomectomy (RALM). Gynecological Endocrinology. 2013;29(1)79-82.

25. Pitter MC, Gargiulo AR, Bonaventura LM. Pregnancy outcomes following robot-assisted myomectomy. Hum Reprod. 2013;28:99-108. 
26. Goldberg J, Pereira L. Pregnancy outcomes following treatment for fibroids: Uterine fibroid embolization versus laparoscopic myomectomy. Curr Opin Obstet Gynecol. 2006;18:402-6.

27. leiomyomata on the results of in-vitro fertilization treatment. Hum Reprod. 1995;10:2576-8.

28. Lonnerfors C, Persson J. Pregnancy following robotassisted laparoscopic myomectomy in women with deep intramural myomas. Acta Obstetricia et Gynecologica Scandinavica. 2011;90(9):972-7.

29. Tusheva OA, Gyang A, Patel SD. Reproductive outcomes following robotic-assisted laparoscopic myomectomy (RALM). Journal of Robotic Surgery. 2013;7(1):65-9.

30. Metwally M, Cheong YC, Horne AW. Surgical treatment of fibroids for subfertility. Review. The Cochrane Collaboration. 2012;11:1-37.

31. Sudik R, Husch K, Steller J. Fertility and pregnancy outcome after myomectomy in sterility patients. European Journal of Obstetrics and Gynecology and Reproductive Biology. 1996;65(2)209-14.

32. Wilcox AJ, Weinberg CR, O’Connor JF. Incidence of early loss of pregnancy. $N$ Engl $J$ Med. 1988;319:189-94.

33. American College of Obstetricians and Gynecologists. Early pregnancy loss. ACOG Practice Bulletin 150. Washington, DC: ACOG; 2015.

34. Pitter MC, Srouji SS, Gargiulo AR. Fertility and symptom relief following robot-assisted laparoscopic myomectomy. Obstetrics and Gynecology International. 2015;2015:1-9.
35. Koo Y, Lee J, Yoo L. Pregnancy outcomes and risk factors for uterine rupture after laparoscopic myomectomy: a single-center experience and literature review. Journal of Minimally Invasive Gynecology. 2015;22(6):1022-8.

36. Harris WJ. Uterine dehiscence following laparoscopic myomectomy. Obstet Gynecol. 1992;80:545-546.

37. Bannerman CG, Gilbert S, Landon MB. Risk of uterine rupture and placenta accreta with prior uterine surgery outside of the lower segment. 2012;120(6):1332-7.

38. Tsuji S, Takahashi K, Imaoka I. MRI evaluation of the uterine structure after myomectomy. Gynecol Obstet Invest. 2006;61:106-10.

39. Barakat EE, Bedaiwy MA, Zimberg S. Roboticassisted, laparoscopic, and abdominal myomectomy: a comparison of surgical outcomes. Obstet Gynecol. 2011;117:256-65.

40. Zhang Y, Hua KQ. Patients' age, myoma size, myoma location, and interval between myomectomy and pregnancy may influence the pregnancy rate and live birth rate after myomectomy. J Laparoendosc Adv Surg Tech. 2014;24(2):95-9.

Cite this article as: Shaia KL, Sekhon LH, Rosen L, Robbins A, Astill N, Ascher-Walsh C. Reproductive outcomes following robotic myomectomy. Int J Reprod Contracept Obstet Gynecol 2017;6:1737-45. 


\section{Appendix A}

\section{Pregnancy after myomectomy questionnaire}

1. What was the main reason you had surgery to have your fibroids removed?

Pain Heavy or Irregular Menses Urinary symptoms Infertility

2. What were secondary reasons you decided to have a myomectomy?

Pain Heavy or Irregular Menses Urinary symptoms Infertility

3. Before your surgery, did you try to get pregnant? (IF NO GO TO 7)

Yes No

4. Before your surgery, did you use any fertility treatments? (IF NO GO TO 6)

Yes No

5. If yes, what treatments did you try?

Clomid IUI Injectables IVF acupuncture other

6. Did you successfully achieve pregnancy and what were the outcomes of those pregnancies, including miscarriages and abortions?

7. Since the surgery have you tried to get pregnant? (IF NO GO TO \#24)

Yes No

8. How long after surgery did you start trying to get pregnant?

$<3$ months 3-6 m 6m-1 yr $>1 \mathrm{yr}>2 \mathrm{yrs}$

9. Since the surgery did you use any fertility treatments?

Yes No 
10. If yes, which ones?

Clomid IUI Injectables IVF acupuncture other

11. Did you successfully get pregnant?

Yes No

12. If yes, was/were the pregnancy/ies spontaneous or a success of your fertility treatment?

Spontaneous Fertility treatment

13. What were the outcomes of those pregnancies, including miscarriages and abortions?

Have you had any spontaneous abortions (miscarriages) since your surgery?

14. If yes, how many miscarriages?

123 More than 3

15. How many weeks pregnant were you at time of miscarriage?

Less than 6 6-10wks 10-14wks 14-20wks >20wks

16. Have you had any elective terminations of pregnancy since your surgery?

Yes No

17. Have you given birth to any children since your surgery?

Yes No

18. If yes, please answer the following

19. How many weeks were you at delivery?

24- $<2828-<3434-<3737-<40>40$ 
20. Did you have a vaginal delivery or c-section?

Vaginal C-section

21. If c-section, did you attempt to have a vaginal delivery?

Yes No

22. If c-section, what was the reason(s) for c-section? Circle all that apply.

Failure to progress in labor Fetal distress Breech

Previous myomectomy elective other

23. Did you experience a complication called uterine rupture or placental abnormalities during your pregnancy or delivery?

Yes No

24. Going back to other reasons why you may have had your surgery... did you have resolution or your symptoms?

No improvement in symptoms

Yes - a small improvement in symptoms

Yes - a significant improvement in symptoms

Yes - complete resolution of symptoms

25. Did you have recurrence of your symptoms?

Yes No

If yes, which symptoms recurred?

Pain/pressure heavy/prolonged/irregular bleeding urinary symptoms

26. How long after surgery did your symptoms return?

Within 6 months 6 month - 1 year 1-2 yrs More than 2 yrs

27. Have you had any other treatments (medical or surgical) for your fibroids since your myomectomy with Dr. AscherWalsh? 